\title{
Nano-copper Oxide as Catalyst for Click Reactions
}

Reda Abdelhady Mustafa ElKhashab ${ }^{1,2^{*}}$, Abdelaziz Ahmed Nayl ${ }^{1,3}$, Elsayed Mohamed Badawy ${ }^{1,2}$ and Tamer Abdelmoemen El Malah, ${ }^{1,4}$

1. Chemistry Department, College of Science, Aljouf University, Sakaka, Kingdom of Saudi Arabia

2. National Center for Clinical and Environmental Toxicology, Faculty of Medicine, Cairo University, Cairo, Egypt

3. Hot Laboratories Centre, Atomic Energy Authority, Cairo, P.O. 13759, Egypt

4. Photochemistry Department, National Research Centre, 33 El Buhouth Street, PO 12622, Dokki, Giza, Egypt

\begin{abstract}
Click reactions are not specific reactions, but they are a way of generating products that follow examples in nature by joining small moieties, with each other producing a huge molecule in a good yield. The mind of that reaction is used in biomolecules synthesis, pharmacological and various biometric applications. The first Click reaction is the Copper compounds-catalyzed reaction of an azide with an alkyne (CuAAC), this copper-catalyzed "click" does not require legands on the metal but the metal oxides also can accelerate the reactions. For enhancement the products of Click reactions we were replacing the copper compounds in a classical reaction by the prepared nanocopper compound (NPs). And measure the consumption of starting material. Behind the evolution is the catalytic effect of nanocopper compounding (NPs) on $\left(\mathrm{H}_{2} \mathrm{O}_{2}\right)$. Owing to the huge surface area of nanocopper compound (NPs), it was found that: the (NPs) can speed up decomposition of $\mathrm{H}_{2} \mathrm{O}_{2}$, also can accelerate the classical click reaction.
\end{abstract}

Key words: Nanocopper oxide, click reactions, catalysis, hydrogen peroxide.

\section{Introduction}

The production of the most industrially important chemicals involves catalysis. Research into catalysis is a major field in applied science and involves many areas of chemistry [1]. A catalyst provides an alternative pathway to the reaction products. The rate of reaction is increased as this alternative route has lower activation energy than the reaction rate in the absence of a catalyst [2]. Many transition metals and transition metal oxides are used in catalysis [3]. The heavy metal oxides are used for their activity in various chemical reactions [4]. It is found the catalytic activity of the oxides is greatly affected by the surface and bulk properties of metal oxides [5]. In parliamentary law to study the catalytic action of different catalysts, decomposition of hydrogen peroxide is applied by different scientists and researcher that for well like indicator reaction [6] as

Corresponding author: Reda Abdelhady Mustafa Elkhashab, assistance professor, main research field: bioinorganic chemistry. the conversion of hydrogen peroxide to water and oxygen are strongly moved by the catalyst. And also the catalytic decomposition of $\mathrm{H}_{2} \mathrm{O}_{2}$ is extensively studied due to its various applications [7-9]. At room temperature the decomposition of hydrogen peroxide is very slow but in the mien of a catalyst decomposition will be triggered. This procedure takes place in homogeneous as well as heterogeneous system [10]. The kinetics of hydrogen peroxide decomposition depend mainly on experimental conditions such as $\mathrm{pH}$, concentrations of hydrogen peroxide, and the catalyst. In the case of heterogeneous catalysis, moreover, the social system and surface properties of catalysts have to be taken into account. Generally speaking, the principal factor determining catalytic efficiency in heterogeneous catalysis is the sufficiently large surface area, which can be achieved by the use of either nanoparticles (NPs), or granular particles with porous character [12]. The decomposition of hydrogen peroxide over a catalyst or an enzyme was studied by several transition metal ions and metal complexes have been used to catalyze this reaction. Metal salts 
and complexes have also been used. However, in most of the studies, it has been observed that single salts, binary or ternary metal complex species have been employed. In the present studies, copper oxide in nano-particles (prepared by reduction of $\mathrm{Cu}^{+2}$ solution) has been used in the kinetic field of the catalytic decomposition of hydrogen peroxide [13]. Comparing with macro-particles of $\mathrm{CuO}$ and the $\mathrm{MnO}_{2}$, they then evaluate the effect of copper oxide (NPs) on Click reaction.

\section{Methods}

\subsection{Preparation of Nanoparticles (NPs) CuO}

The all chemicals were analytical grade and used as it is without any further purification. Double distilled water was used during the preparation of solutions and washings of the catalyst nanoparticles (NPs). All glasswares used were made up of Pyrex; $\mathrm{CuO}$ nanoparticles (NPs) were prepared by precipitation method in aqueous solution. By using cupric acetate $\left.\left[\mathrm{Cu}\left(\mathrm{C}_{2} \mathrm{H}_{3} \mathrm{O}_{2}\right) \cdot 2 \cdot \mathrm{H}_{2} \mathrm{O}\right)\right]$ (98\%, Sigma-Aldrich) as a starting material and sodium hydroxide $(\mathrm{NaOH})(>$ $98 \%$, China) as a reducing agent. Was also used to adjust the $\mathrm{pH}$ and accelerate the reduction reaction in water. In detail, use $600 \mathrm{~mL} 0.2 \mathrm{M}(39.93 \mathrm{~g} / \mathrm{L})$ of cupric acetate solution with about $2 \mathrm{~mL}$ of glacial acetic acid $\left(\mathrm{C}_{2} \mathrm{H}_{4} \mathrm{O}_{2}\right)$ (Sigma-Aldrich). Were added into a round-bottomed flask and heated to boiling with stirring. After boiling, add, $30 \mathrm{~mL}$ of $6 \mathrm{M}(240 \mathrm{~g} / \mathrm{L})$ of $\mathrm{NaOH}$ ( $>98 \%$, China) into the flask. The semblance of the solution turned from rich down to black immediately, and a black suspension formed with time. The reaction was carried out under stirring and boiling for approximately $2.5 \mathrm{~h}$. The mixture was cooled to room temperature and centrifuged. And then, a $\mathrm{CuO}$ precipitate was obtained. The precipitates were filtered and washed with distilled water and absolute ethanol for several times. The resulting product was dried (at $60{ }^{\circ} \mathrm{C}$ for $6 \mathrm{~h}$ ) to obtain the dry powder of CuO nanoparticles (NPs) [14].

\subsection{Characterization of Nanocopper Oxide (NPs)}

UV-visible spectroscopy is a inexpensive tool for confirming the formation of the syntheses of the copper oxide nanoparticles (NPs). As shown in the Fig. 1a broad absorption peak was observed at around 350 $\mathrm{nm}$, which is an indication of the formation of of the copper oxide nanoparticles [15].

\subsection{Characterization of Nanocopper Oxide (NPs)}

The morphology of as prepared particles was studied by SEM (scanning electron microscopy) and

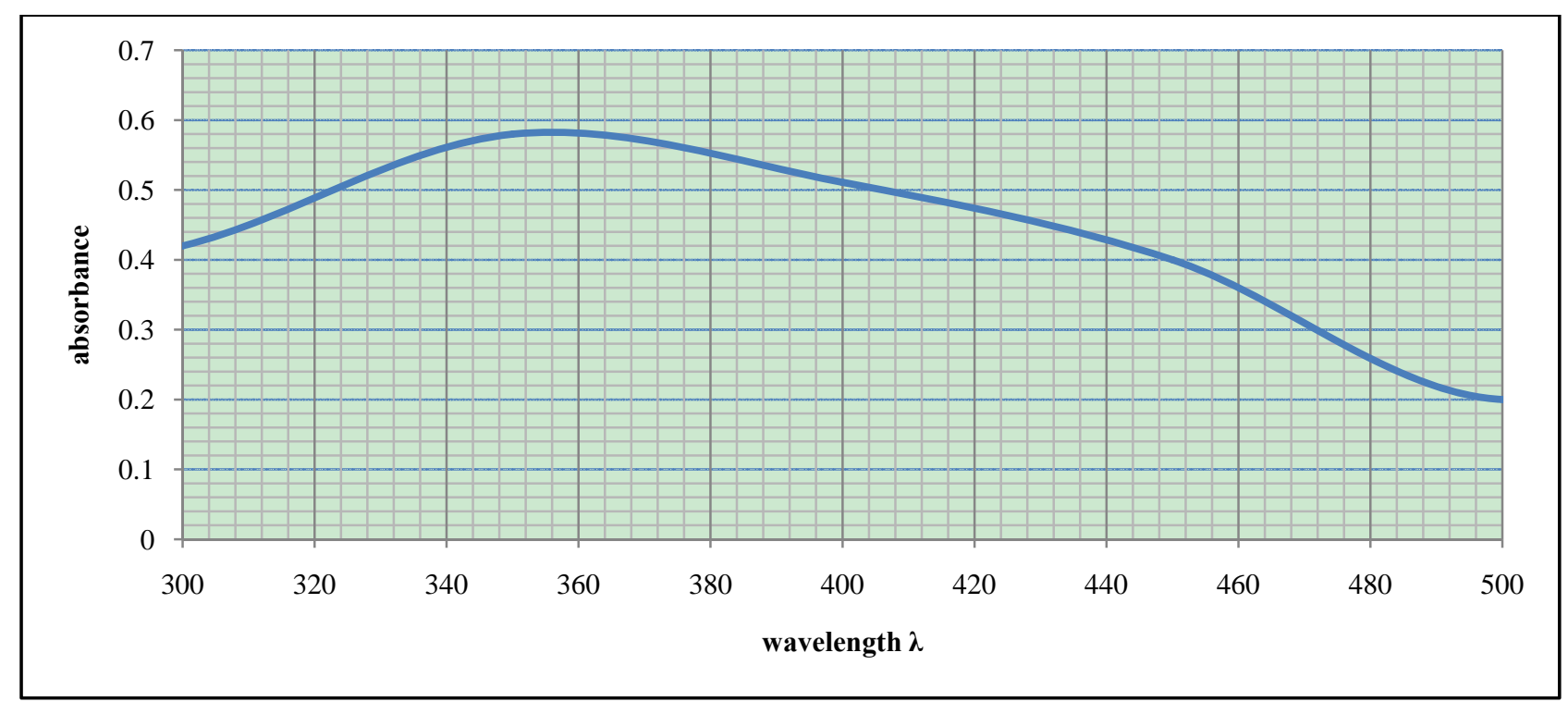

Fig. 1 UV-visible spectrograph for NPs copper oxide. 


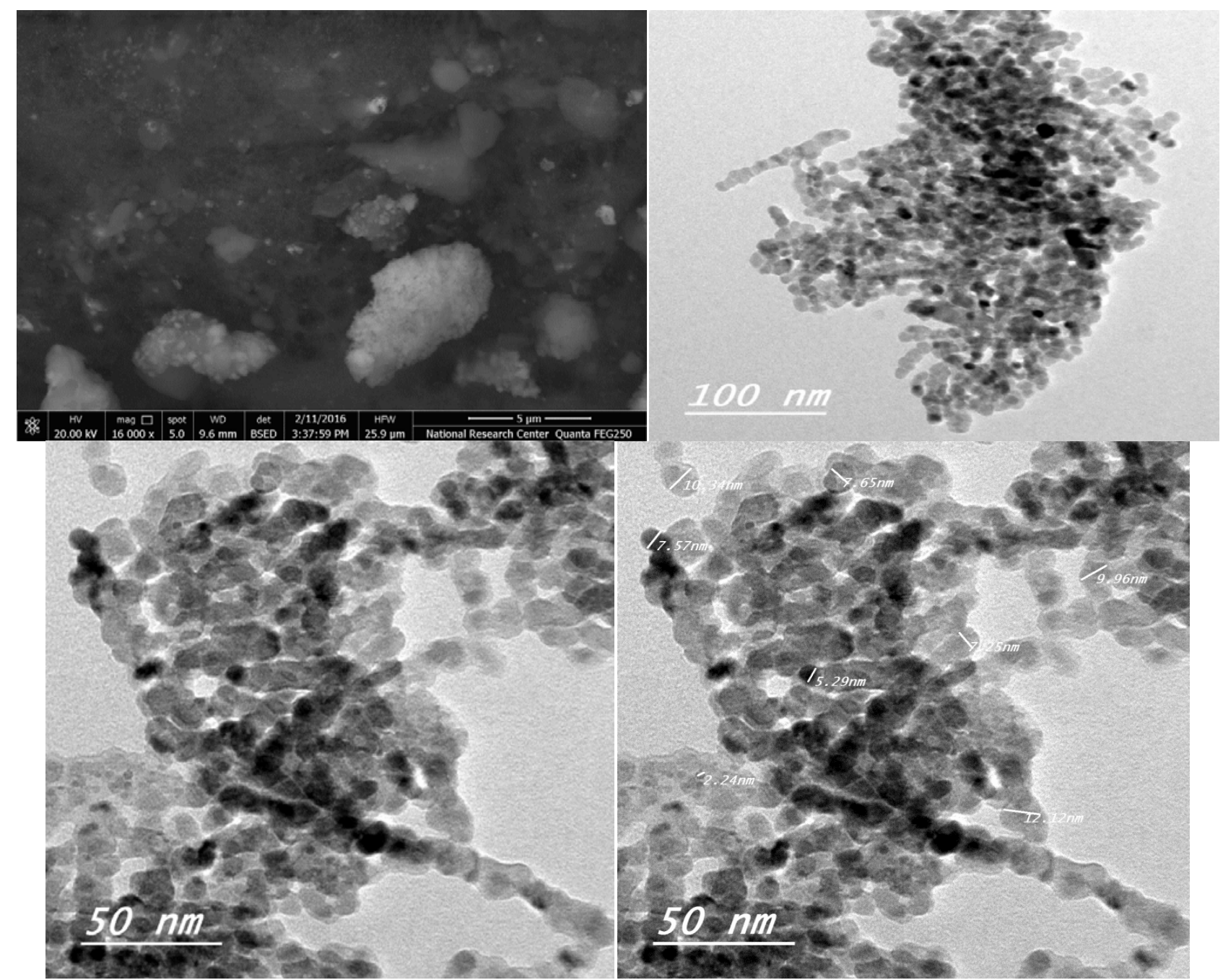

Fig. 2 The SEM and TEM for the prepared nanoparticles (NPs) copper oxide.

TEM (transmission electron microscopy) which indicate the photographs from SEM indicates agglomerated particles at five micron size. By applying a TEM technique Fig. 2 appears more distinct round particles that formed NPs of copper oxide, with diameter size varying between 2.24 and $12.12 \mathrm{~mm}$ (Fig. 2).

\subsection{Kinetic Measurements of Catalytic Decomposition of Hydrogen Peroxide}

The decomposition of hydrogen peroxide was studied in the presence of $\mathrm{MnO}_{2}$, (NPs) nanocopper oxide and macroparticles copper oxide where $\mathrm{MnO}_{2}$ used as standard catalyst. As $\mathrm{H}_{2} \mathrm{O}_{2}$ is sensitive to light, therefore, all the experiments were performed in the absence of light by wrapping the black paper around the flasks to minimize the error. The method applied was the same as reported in the literature. The amount of solid catalyst (NPs $\mathrm{CuO}, \mathrm{CuO}, \mathrm{MnO}_{2}$ ) used in each experiment was $2.0 \mathrm{~g} / \mathrm{L}$. The concentration of $\mathrm{H}_{2} \mathrm{O}_{2}$ was adjusted to $0.02 \mathrm{~mol} \cdot \mathrm{dm}^{-3}$ in all cases. The content of the vessel was stirred uniformly by means of a magnetic stirrer using Teflon coated magnetic bar. Later on an interval of $5 \mathrm{~min}$ about $5 \mathrm{~mL}$ of the reaction mixture was taken back from the double walled vessel and was quickly added to $5 \mathrm{~mL}$ of ice cooled $5 \mathrm{~mol} \cdot \mathrm{dm}^{-3} \mathrm{H}_{2} \mathrm{SO}_{4}$ solutions. The solid was separated from a mixture by centrifugation, and the separator was then titrated against 0.02 male $\mathrm{dm}^{-3}$ $\mathrm{KMnO}_{4}$ solution. From this, the quantity of the decomposed $\mathrm{H}_{2} \mathrm{O}_{2}$ was estimated. In all cases, blank 
experiments were also performed in the absence of the solid in order to investigate the effect, if any of the experimental conditions on the decomposition of $\mathrm{H}_{2} \mathrm{O}_{2}$. By drawing the data for the three kinds of catalysts at specific concentrations (weight of catalysts $\mathrm{H}_{2} \mathrm{O}_{2}$ ), we obtained a graph (Fig. 3), showing the relation between the catalysts kind with percentage decomposed from $\mathrm{H}_{2} \mathrm{O}_{2}$ by the interval time. The times of complete decomposition are obtained by extra plotation of the lines in graph until the time axis (x axis), where the time of complete dissociation of $\mathrm{H}_{2} \mathrm{O}_{2}$ can be ordered as using $\mathrm{MnO}_{2}$, NPs CuO takes less time than macroparticles $\mathrm{CuO}$, equally it can say NPs $\mathrm{CuO}$ is more reactive than macr $\mathrm{CuO}$ in dissociation of $\mathrm{H}_{2} \mathrm{O}_{2}$.

\subsubsection{General Click Chemistry Procedure}

Click chemistry has been utilized for the preparation of new tris(triazolyl)triazines containing aliphatic and polar side chains through coupling of 2,4,6-triethynyl-[1,3,5] triazines, with substituted aromatic azides. Using copper sulphate as catalyst to compare the effect of nanoform of copper oxide (NPs), the authors replace copper sulphate which already used by nanoform of copper oxide (NPs).

\subsubsection{Click Reaction}

That carried out by adding 1,3,5 triazine 2,4,6-triethynyl to aryl azide, sodium ascorbate, TBTA, and a solvent mixture of $\mathrm{H}_{2} \mathrm{O} / \mathrm{tBuOH} / \mathrm{CH}_{2} \mathrm{Cl}_{2}$. The flask was evacuated and flushed with argon. $\mathrm{CuSO}_{4} \cdot 5 \mathrm{H}_{2} \mathrm{O}$ was added and the mixture was stirred for 2 days at room temperature in the dark. In the event of a precipitate appearing, additional $\mathrm{CH}_{2} \mathrm{Cl}_{2}$ was added. After consumption of 1,3,5 triazine 2,4,6-triethynyl as indicated by TLC monitoring, after purification and drying the target compound was obtained with column chromatography.

\subsubsection{Click Reaction with NPs}

The authors replace copper sulphate which already used in click reaction as catalyst, replaced by copper oxide in nanoform (NPs), and then flow the consumption of starting materials in these two expermints (when use copper sulphate, and when use copper oxide nanoform (NPs). that by thin layer chromatographic method.

From Fig. 4 the complete consumption of starting material (1,3,5 triazine 2,4,6-triethynyl) appears after

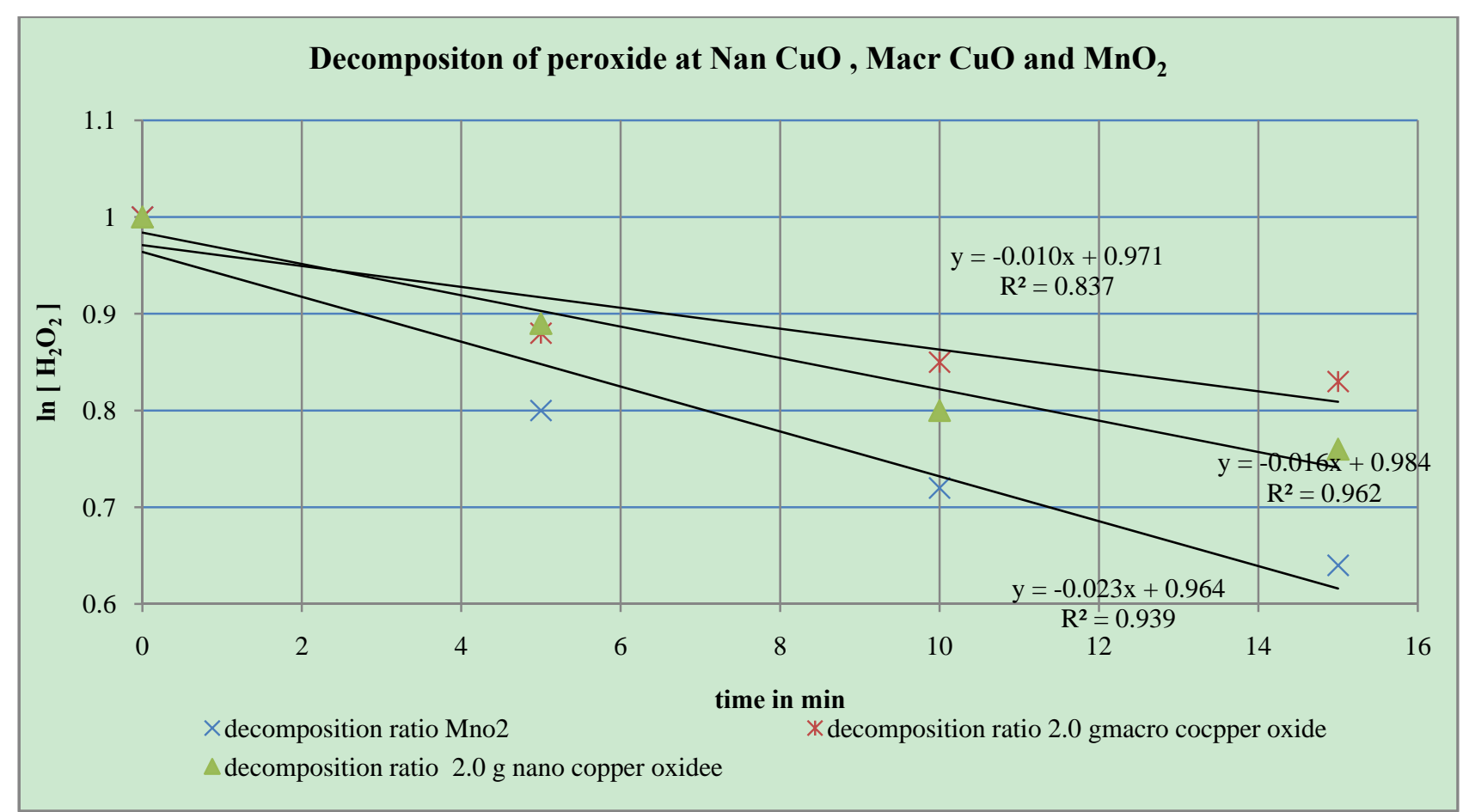

Fig. 3 Decomposion of hydrogen peroxide as $\mathrm{NPs} \mathrm{CuO}, \mathrm{CuO}$, and $\mathrm{MnO}_{2}$. 


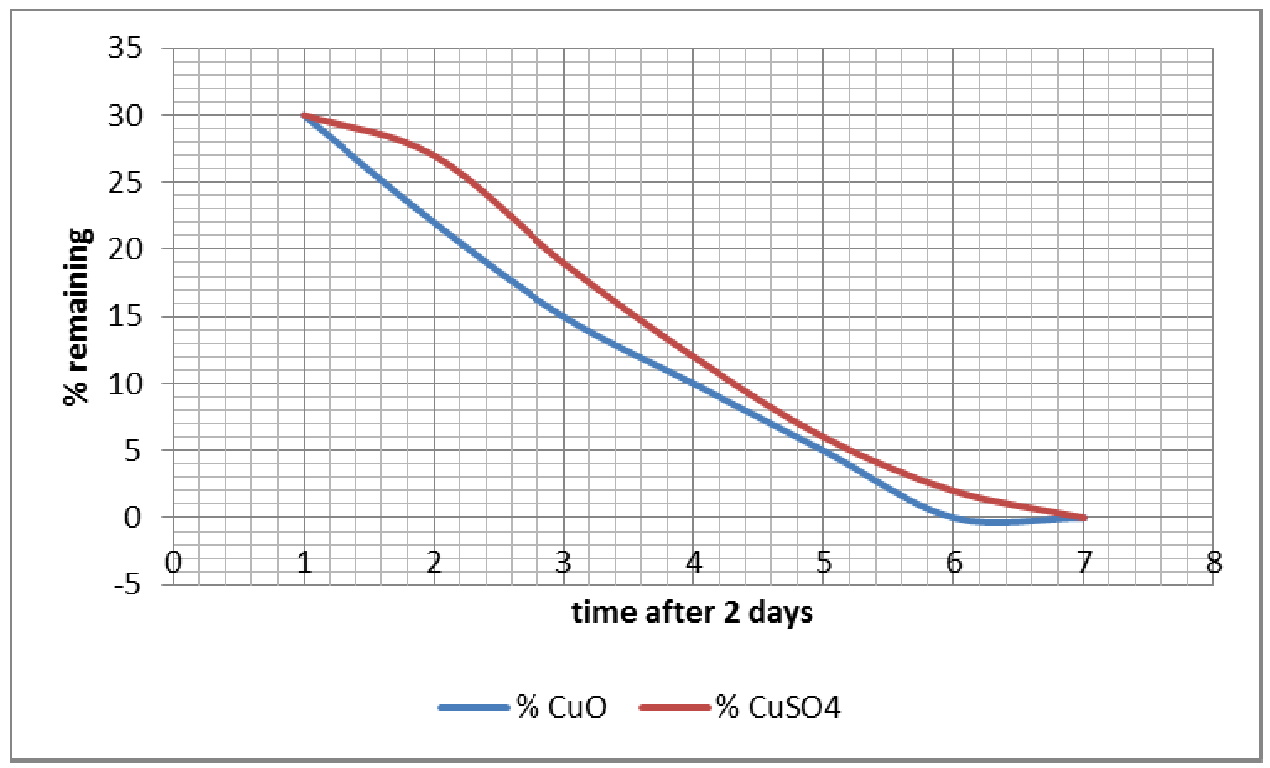

Fig. 4 Consumption of 1,3,5 triazine 2,4,6-triethynyl (starting material) with time.

$54 \mathrm{~h}$, by useing NPs CuO, while it appears at $55 \mathrm{~h}$ by using a copper sulfate. That indicates the NPs copper oxide accelerate the reaction as starting material consume early than reaction incorporate by copper sulfate.

\section{Results and Discussion}

Transition metals and their compounds function as catalysts; either because of their ability to change oxidation state or, to adsorb reactant substances onto their surface and activate them in the process. The decomposition of hydrogen peroxide was studied by many researchers for several transition metal and metal oxides. But the most studies may use an expensive an metal or metal oxides, this metal has several stable oxidation states, but in our research use a cheap copper oxide in NPs nanoparticles forms to avoid the limitation of its oxidation state. And consequently we use the NPs of $\mathrm{CuO}$ in Click reaction instead of $\mathrm{Cu}$ cations as Copper sulphate. By flow the time of consumption for starting material by TLC we notice that: on using NPs we need shorter time than using $\mathrm{Cu}$ cations from copper sulpahe.

\section{Acknowledgement}

The authors acknowledge the Aljouf University for financial support of this work (project number 35/338).

\section{References}

[1] Anastas, P. T. and Kirchhoff, M. M. 2002. "Origin, Current Status, and Challenges of Green Chemistry.” Acc. Chem. Res. 35: 686-94.

[2] R\&D Magazine. 2005. "Recognizing the Best in Innovation: Breakthrough Catalyst.”

[3] Sabu, K. R. P. and Rao, K. V. C. 1991. "The Acidic Property and Catalytic Activity of $\mathrm{MoO}_{3}-\mathrm{SiO}_{2}-\mathrm{Al}_{2} \mathrm{O}_{3}$." Bulletin of the Chemical Society of Japan 64 (6): 1926-32.

[4] Rani, A., Prasad, D. S. N., Bhargava, R. and Gupta, K. S. 1991. "Dynamics of Autoxidation of Aqueous Sulfur Dioxide in Aqueous Suspensions of Cadmium Oxide.” Bulletin of the Chemical Society of Japan 64: 1955-61.

[5] Kwan, W. and Voelker, B. M. 2002. "Decomposition of Hydrogen Peroxide and Organic Compounds in the Presence of Dissolved Iron and Ferrihydrite.” Environmental Science and technology 36 (7): 1467-76.

[6] Akhtar. K. 2009. "Decomposition of Hydrogen Peroxide by Nickel Oxide Powders." Journal of the Chemical Society of Pakistan 31: 59-66.

[7] Gurol, M. D. and Lin, S. S. J. 2002. "Hydrogen Peroxide/Iron Oxide-Induced Catalytic Oxidation of Organic Compounds.” AdV. Oxid. Technol. 5: 147-54.

[8] Perathoner, S. and Centi, G. 2005. "WHPCO (Wet Hydrogen Peroxide Catalytic Oxidation) of Organic Waste in Agro-food and Industrial Streams.” Top. Catal. 33: 207-24. 
[9] Watts, R. J., and Teal, A. L. J. 2005. "Chemistry of Modified Fenton's Reagent (Catalyzed $\mathrm{H}_{2} \mathrm{O}_{2}$ Propagations-CHP) for In Situ Soil and Groundwater Remediation.” EnViron. Eng. Reston Va 131: 612-22.

[10] Vielstich, W. 1980. "Relation between the Activity of Electrodes from Various Carbonaceous Meterials for Reduction of Oxygen to Hydrogen Peroxide and Concentration of Their Paramagnetic Centers." Collection of Czechoslovak Chemical Communications 45: 3249-53.

[11] Larpent, C. and Patin, H. J. 1992. "Oxidation of Alkanes with Hydrogen Peroxide Catalysed by Iron Salts or Iron Oxide Colloids in Reverse Microemulsions.” Mol. Catal. 72: 315-29.

[12] Hunag, H. H., Lu, M. C. and Chen, J. N. 2001. “Catalytic Decomposition of Hydrogen Peroxide and 2-chlorophenol with Iron Oxides.” Water Res.: 2291-9.

[13] Sayed, M., Badawy, R. A., El. Khashab, A. A. 2015. "Synthesis, Characterization and Catalytic Activity of $\mathrm{Cu} / \mathrm{Cu}_{2} \mathrm{O}$ Nanoparticles Prepared in Aqueous Medium.” Bulletin of Chemical Reaction Engineering \& Catalysis 10 (2): 169-74.

[14] Maqusood, A., Hisham, A., Alhadlaq, M. A., Majeed, K., Ponmurugan, K. and Naif, A. A. 2014. "Synthesis, Characterization, and Antimicrobial Activity of Copper Oxide Nanoparticles.” Journal of Nanomaterials Volume Article ID 637858.

[15] Hamid, R., Ghornani, I. F. and Ali, A. F. 2015. "Biosynthesis of Copper Oxide Nanoparticles using Extract of E.coli oriental.” Journal of Chemistry 31 (1): 515-7. 